\title{
Sleepiness and impulsivity: Findings in non-treatment seeking young adults
}

\author{
JON E. GRANT ${ }^{1 *}$ and SAMUEL R. CHAMBERLAIN ${ }^{2,3}$ \\ ${ }^{1}$ Department of Psychiatry \& Behavioral Neuroscience, Pritzker School of Medicine, University of Chicago, Chicago, IL, USA \\ ${ }^{2}$ Department of Psychiatry, University of Cambridge, Cambridge, UK \\ ${ }^{3}$ Cambridge and Peterborough NHS Foundation Trust, Cambridge, UK
}

(Received: October 27, 2017; revised manuscript received: May 30, 2018; accepted: June 11, 2018)

\begin{abstract}
Background and aims: Although inadequate sleep has been linked to problematic behaviors, such as poor impulse control and emotion dysregulation, little research interest has been the role of sleep and sleep deprivation on impulsive behaviors in young adults. To further examine the relationship of sleepiness to impulsivity and impulsive behaviors, this study was designed to collect data on sleepiness, and a range of impulse control disorders and cognitive measures. Methods: Young non-treatment-seeking adults were recruited from two US cities and completed a screening form for sleepiness, along with demographic, clinical, and cognitive measures relevant to impulsivity. Relationships between these explanatory variables and total sleepiness scores were analyzed using partial least squares. Significant explanatory variables were identified $(p<.05$, bootstrap). Results: Higher levels of sleepiness were significantly associated with higher ADHD symptoms, gambling disorder symptoms, Internet addiction symptoms, and personality-related impulsiveness. Sleepiness was also associated with set-shifting errors, and with gambling more points (abnormal decision-making), but not with significant impairment in response to inhibition, or other aspects of decision-making. Conclusions: This study confirms a cross-sectional relationship between sleepiness and a range of impulsive measures at the level of behavior (ADHD, gambling, and Internet addiction) and personality traits. Longitudinal research would be required to explore causal mechanisms and the direction of any such effects. Screening for such mental health diagnoses in people with sleep problems may be valuable, as enquiring patients with such impulsive symptoms about sleep, in order to maximize quality of life.
\end{abstract}

Keywords: sleep, impulsivity, cognition

\section{INTRODUCTION}

Early adulthood is a developmental period associated with impulsive behaviors (Casey, Heller, Gee, \& Cohen, 2017; Kubera et al., 2018). Peer groups, brain development, and environmental and genetic influences have all been implicated in the frequency and intensity of impulsive behaviors in this age group (Quinn, Stappenbeck, \& Fromme, 2011). One area that has merited media attention but has received little research interest has been the role of sleep and sleep deprivation on impulsive behaviors in young adults.

Inadequate sleep has been linked to problematic behaviors, such as poor impulse control and emotion dysregulation (Beebe, 2011). Specifically, young adults appear most vulnerable to negative sleep-related outcomes, possibly due to a complex interaction between sleep deprivation and the lack of maturation of inhibitory brain circuitry in this age cohort (Chee \& Choo, 2004; Drummond et al., 2000). Further support for this link between sleep deprivation and impulsivity comes from studies that have found impaired response inhibition, risky decision-making, and increased risk-seeking all associated with sleep deprivation in adulthood, including in young adults (Frings, 2012; Kendall, Kautz, Russo, \& Killgore, 2006; Killgore, Balkin, \& Wesensten, 2006; Venkatraman, Chuah, Huettel, \& Chee, 2007). Overall, these studies suggest that sleepiness (either produced by prolonged and continual wakefulness, fatigue, or accumulated sleep debt) is associated with impulsivity on some neuropsychological tasks.

In terms of impulsive behaviors, sleep problems have been associated with drug and alcohol use, including in younger people (Schierenbeck, Riemann, Berger, \& Hornyak, 2008; Wong, Brower, \& Zucker, 2009) and with formal substance-use disorders (Breslau, Roth, Rosenthal, \& Andreski, 1996; Stein \& Friedmann, 2005). In the case of one of the more extreme versions of impulsivity, attentiondeficit hyperactivity disorder (ADHD), sleep has been examined as a potential contributor to the issue of impulsivity (Jan, Yang, \& Huang, 2011). In a large study $(n=112)$ of forensic psychiatric adult inpatients with antisocial or borderline personality disorder, both poor sleep quality and chronic insomnia were significantly associated with elevated self-reported impulsivity (Van Veen, Karsten, \& Lancel, 2017). These studies suggest that

\footnotetext{
* Corresponding author: Jon E. Grant, JD, MD, MPH; Department of Psychiatry \& Behavioral Neuroscience, Pritzker School of Medicine, University of Chicago, 5841 S. Maryland Avenue, MC-3077, Chicago, IL 60637, USA; Phone: +1 773834 1325; Fax: +1 773834 6761; E-mail: jongrant@uchicago.edu
}

This is an open-access article distributed under the terms of the Creative Commons Attribution-NonCommercial 4.0 International License, which permits unrestricted use, distribution, and reproduction in any medium for non-commercial purposes, provided the original author and source are credited, a link to the CC License is provided, and changes - if any - are indicated. 
problematic sleep is somehow related to important aspects of impulsive behavior.

To further examine the relationship of sleepiness to impulsivity and impulsive behaviors, this study was designed to collect data on sleepiness (as defined in Johns, 1991), amount of sleep received, and a range of impulse control disorders and cognitive measures. In order to extend beyond existing work, this study design incorporated a broad range of impulsivity measures not typically included in prior sleepiness research, such as screening for impulse control disorders and gambling disorder, and inclusion of both personality- and cognitive-related impulsivity outcome measures. Furthermore, we used a powerful statistical technique, such as partial least squares (PLS), which is ideally suited for situations involving variables that may be correlated. We hypothesized that those with greater levels of daytime sleepiness would report more impulse control disorders and exhibit greater levels of impulsivity on standardized measures.

\section{METHODS}

A total of 373 young adults (age range: 18-29 years) from two midwestern metropolitan areas voluntarily participated in a study on impulsivity. Participants were recruited through media advertisements and by word of mouth. Study subjects completed their visits within offices in the campuses of the Universities of Chicago and Minnesota. Participants were compensated with $\$ 50$ gift cards to a nationwide department store. Study visits lasted approximately for $2 \mathrm{hr}$ and participants were allowed to take breaks as necessary. Two trained raters supervised by a boardcertified psychiatrist carried out the assessments.

\section{Subjects}

The study requirements included the participants to have gambled five times in the past 12 months (a proxy for some level of baseline impulsivity), being within ages 18-29 years, being able to understand and sign the study consent form, and able to perform the study procedures.

\section{Clinical assessments}

The Epworth Sleepiness Scale (ESS; Johns, 1991), a reliable and valid scale of sleepiness, was used as the measure of subjects' sleepiness. The ESS poses eight hypothetical situations, in which the test taker rates in terms of how likely they would be to fall asleep in them: (0) no, (1) slight, (2) moderate, and (3) high chance of dozing. Total scores range from 0 to 24 . Scores less than or equal to 9 indicate a person who has an average amount of daytime sleepiness or is unlikely to be abnormally sleepy; a score of 10-15 indicates a tendency to be excessively sleepy depending on the situation; whereas a score of 16-24 indicates excessive sleepiness and the person should consider seeking medical attention (Johns, 1991).

Raters assessed each participant using the MiniInternational Neuropsychiatric Interview (M.I.N.I.; Sheehan et al., 1998), and the Minnesota Impulse Control Disorder
Interview (M.I.D.I.; Chamberlain \& Grant, 2018); the former screens for mainstream mental disorders (e.g., depression and anxiety), whereas the latter screens for impulse control disorders (e.g., trichotillomania and kleptomania). Both instruments were designed as short structured clinical interviews for the assessment of mental disorders in research and clinical settings. Both have good-excellent test-retest and interrater reliability.

To assess personality-related impulsivity, participants completed the Barratt Impulsiveness Scale Questionnaire version 11 (BIS-11; Patton, Stanford, \& Barratt, 1995), which is a 30 -item instrument that uses a 4-point scale for each response (responses are: rarely/never, occasionally, often, or almost always/always). The BIS-11 responses are used to generate three subscores on the basis of previous factor analysis, such as motor, non-planning, and attentional impulsivity.

The Adult ADHD Self-Report Scale (v1.1), a six-item measure, was used to quantify ADHD symptomatology (Kessler et al., 2005, 2007). Problem gambling behaviors were quantified using the Structured Clinical Interview for Gambling Disorder (SCI-GD; Grant, Steinberg, Kim, Rounsaville, \& Potenza, 2004) (adapted from Grant et al., 2004 to consider DSM-5 gambling disorder) and problem Internet behaviors with Young's (2009) Internet Addiction Diagnostic Test.

\section{Cognitive assessment}

Cognitive testing was undertaken using computerized paradigms from the Cambridge Neuropsychological Test Automated Battery. Participants completed cognitive testing in a quiet room, using a touch-screen computer.

Decision-making was examined using the Cambridge Gamble Task (CGT; Rogers et al., 2003). Participants were informed that for each trial, the computer had hidden a "token" inside one of 10 boxes shown on the screen. These boxes were each either red or blue, and the participant indicated whether they felt the token would be hidden behind a red or a blue box. After making this judgment, participants gambled a proportion of their points on whether their color choice was correct. The key outcome measures were quality of decision-making (the proportion of trials where the volunteer chose red when red boxes were in the majority and vice versa - i.e., made the logical color choice), proportion bet, and risk adjustment.

We assessed response inhibition using the Stop-Signal Task (SST; Aron, Fletcher, Bullmore, Sahakian, \& Robbins, 2003), a paradigm in which the participant viewed a series of directional arrows appearing one per time on-screen, and made quick motor responses depending on the direction of each arrow (left button for a left-facing arrow and vice versa). On a subset of trials, an auditory stop-signal occurred (a "beep") to indicate that response suppression was required for the given trial. The main outcome measure of the SST is the stop-signal reaction time (SSRT), which is an estimate of the time taken by the given volunteer's brain to suppress a response that would normally be undertaken.

Set-shifting was measured using the intradimensional/ extradimensional set-shift task (IED; Pantelis et al., 1999). This task, derived from the Wisconsin Card Sorting Task, 
quantifies several aspects of rule learning and flexible behavior. Volunteers choose from two stimuli presented on the screen on each trial, and attempt to discover an underlying rule governing which stimulus is "correct" (based on simple feedback provided by the computer). Once the volunteer has learned a given rule, the task then changes the rule. The main outcome measure on the task is the total number of errors made, adjusted for stages that were not attempted.

\section{Data analysis}

To identify demographic, clinical, and cognitive measures associated with variation in sleepiness, we utilized the statistical technique of PLS (Abdi \& Williams, 2013; Höskuldsson, 1988). PLS is a multivariate, iterative technique that constructs one or more latent factors (referred to as PLS components) that optimally explain variation in $X$ and $Y$. The $Y$ variable was a total score on the ESS and $X$ variables were as follows: age, gender, education level, employment status, presence of impulse control disorder(s) (on the M.I.D.I.), presence of mainstream mental disorder(s) (on the M.I.N.I.), disordered gambling symptoms (criteria endorsed on the SCI-GD), ADHD symptoms, Barratt impulsivity (attentional, motor, and non-planning), SSRT, quality of decision-making (CGT), overall proportion of points gambled (CGT), risk adjustment (CGT), and total set-shifting errors (IED). Unlike traditional regression, PLS is suitable in situations in which variables are correlated with each other, as was expected here. Analysis was conducted using JMP Pro software version 13.0. Any missing data points were imputed automatically by JMP software using study means. The PLS model was fitted using leave-one-out crossvalidation (non-linear iterative PLS algorithm), and the optimal number of latent factors was selected by minimizing the predictive residual sum of the squares. $X$ variables that are significantly contributing to the model (i.e., explaining significant variance in disease severity) were identified on the basis of $95 \%$ confidence intervals for bootstrap distribution of the standardized model coefficients not crossing zero $(N=2,500$ bootstraps).
Our above analysis of ESS as a continuous variable was also complemented by a secondary more traditional categorical approach, which is reported in the Supplementary Material. Subjects were classified a priori into one of the following three groups based on overall ESS score: average amount of daytime sleepiness or is unlikely to be abnormally sleepy (score of 9 or less) ("normal"), a tendency to be excessively sleepy depending on the situation (score of 10-15) ("situational"), and excessive sleepiness and the person should consider seeking medical attention (score of 16-24) ("excessive"). Analysis of variance approach was used to explore clinical and cognitive differences between the identified gambling subtypes. Where there was a main effect of group on a given variable, this was further explored using $t$-tests. Alternative non-parametric tests were used where appropriate. Statistical significance was defined as $p<.05$, two-tailed.

\section{Ethics}

All study procedures were carried out in accordance with the Declaration of Helsinki. The institutional review boards of the Universities of Chicago and Minnesota approved the study and the consent statements. Participants provided written informed consent.

\section{RESULTS}

The overall sample of 373 participants had mean (standard deviation) age of 22.5 (3.6) years and were $62.5 \%$ male. PLS yielded an optimal one factor solution, which explained $18.1 \%$ of variance in $X$ variables and $13.0 \%$ of variance in ESS total scores. The standardized model coefficients for the explanatory variables are shown in Figure 1. It can be seen that higher total ESS scores were significantly $(p<.05$, bootstrap) predicted by presence of any M.I.N.I. mental disorder, gambling disorder number of criteria met, ADHD symptoms, problematic Internet use, Barratt impulsivity (all three subscales individually), overall proportion of points

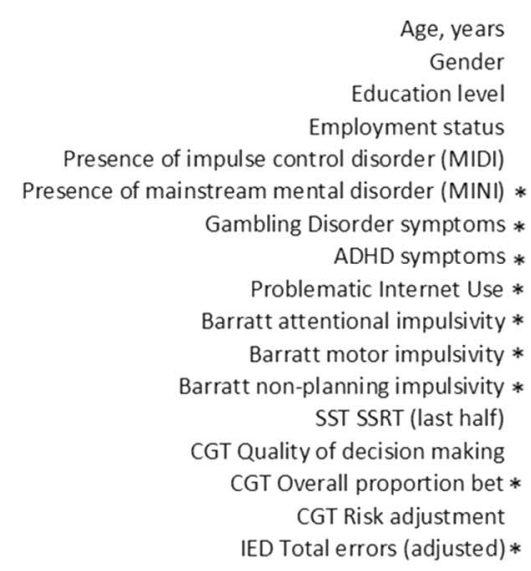

Figure 1. Results from PLS model coefficients relating explanatory variables to ESS total scores. *Significant non-zero coefficient by bootstrap $(p<.05)$

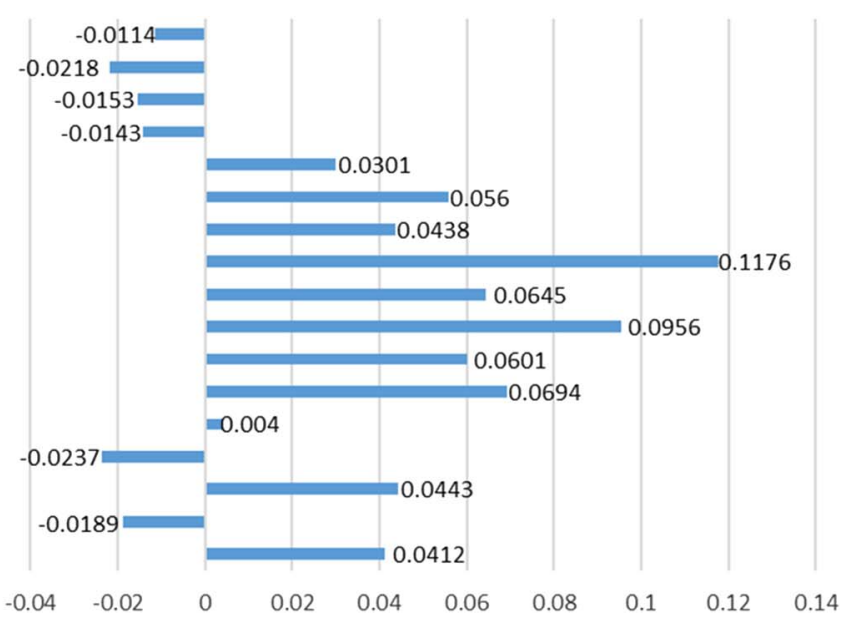


gambled on the CGT, and IED number of errors made. Secondary analysis using a traditional categorical approach to ESS yielded a similar profile of results, except that the effect of group on IED total errors was not significant (Supplementary Material).

\section{DISCUSSION}

In this study, we examined impulsive behaviors and cognitive domains relevant to impulsivity, in a non-treatment seeking sample of young adults with varying levels of sleepiness. We hypothesized that problems with sleepiness would be associated with a greater likelihood of having impulse control disorders and exhibiting impulsivity on a variety of cognitive measures, as well as on the BIS- 11 . Based on the results of the analysis we found that this hypothesis was partly supported. Higher levels of sleepiness were significantly associated with several types of impulsive symptoms (gambling disorder, ADHD, and problematic use of the Internet), with questionnaire-rated impulsivity (all three Barratt questionnaire subscales), and with gambling more points on the computerized decision-making task. This suggests a fundamental relationship between sleepiness and multiple (but not all) types of impulsivity in this nontreatment seeking sample, which may reflect either causative mechanisms (i.e., impulsivity causes sleepiness) or just an association (e.g., impulsive individuals are more prone to sleep disturbance).

Greater problems with sleepiness were significantly associated with higher rates of impulsive behaviors including as gambling, ADHD, or Internet addiction. Simultaneously, sleepiness scores were not associated with having other impulse control disorders such as kleptomania, compulsive sexual behavior, or compulsive buying (as indexed by the M.I.D.I.). Our findings are broadly consistent with previous research. As in this study, Parhami et al. (2012) found significantly higher ESS scores associated with more gambling disorder symptoms. Subjective reports of sleep disturbance have been widely reported in ADHD, both in childhood (Corkum, Tannock, \& Moldofsky, 1998) and in adulthood (Instanes, Klungsoyr, Halmoy, Fasmer, \& Haavik, 2018). Some studies have also examined sleepiness in the context of Internet Addiction. In a sample of high school students in South Korea, excessive daytime sleepiness occurred in $37.7 \%$ of individuals with Internet addiction (Choi et al., 2009). In an another study, adolescents with higher Internet addiction scores reported going to bed later at night, needing more time to get to sleep, and more night awakenings (Ekinci, Celik, Savas, \& Toros, 2014). Our finding that certain behaviors were more likely associated with sleepiness highlights the importance of careful screening for sleep disorders and impulsive disorders in clinical practice.

In terms of general impulsiveness not related to a disorder, we found that sleepiness problems were associated with higher scores on all three subscales of the BIS-11. The BIS-11 captures a range of impulsive tendencies that are related to day-to-day life. Contrary to our expectation, we did not find that the study groups differed significantly for SSRT, which is a laboratory-based measure of prepotent response inhibition (SST). Factor analysis suggests that Barratt questionnaire and stop-signal impulsivity are in fact somewhat dissociable, reflecting latent factors of impulsive action and impulsive personality traits, respectively (MacKillop et al., 2016). Thus, our results demonstrate an association between sleep problems and personality traits of impulsiveness, rather than impulsive action per se. In addition to sleepiness being associated with higher Barratt impulsivity, sleepiness was also associated with gambled significantly more points on the CGT, and with more set-shifting errors on the set-shift task. Other aspects of decision-making on the CGT were not statistically significantly associated with sleepiness. Interestingly, preclinical work found that sleep fragmentation led to poorer sleep in rats (McCoy et al., 2007). One previous human study using the Iowa Gambling Task, which bears parallels to the CGT, found that after $49.5 \mathrm{hr}$ of continual wakefulness, healthy adults began to make increasingly risky and disadvantageous choices (Killgore et al., 2006). It would be interesting in future work to examine other measures of impulsivity, such as reflection impulsivity and monetary discounting tasks (MacKillop et al., 2016).

Several limitations should be considered regarding this study. We explored relationships between sleepiness and various impulsivity measures, but due to the cross-sectional nature of the research, causality cannot be inferred. We used a subjective scale for sleepiness and so findings with objective sleep/arousal measures may differ. The nature of the study design may introduce response bias (convenience sampling). Finally, we focused on a restricted range of cognitive measures and so there may be associations between sleepiness and other aspects of impulsivity (and compulsivity) not measured here.

In summary, this study found that excessive sleepiness was associated with maladaptive gambling, ADHD, and Internet addiction symptoms; higher scores on personality impulsivity; and abnormalities of decision-making (gambling more points) and set-shifting (more errors). Future work would be valuable to clarify whether these relationships are causal, ideally using a larger sample size. Based on these findings, it would seem important to enquire people presenting with sleepiness whether they have impulsive symptoms and vice versa, so that treatment approaches can be optimized for both types of impairing problems.

Funding sources: This research is supported by a Center for Excellence in Gambling Research grant by the National Center for Responsible Gaming (NCRG) to Dr. JEG and a Wellcome Trust Clinical Fellowship to Dr. SRC (UK; reference 110049/Z/15/Z).

Authors' contribution: Dr. JEG designed the study and conducted the data collection. Both authors contributed to data analysis and writing of the manuscript. They take full responsibility for the contents of the manuscript and the integrity of the data.

Conflict of interest: Dr. JEG is chair of the Scientific Advisory Board of the TLC Foundation for BFRBs and 
currently received funding from its BFRB Precision Medicine Initiative. In addition, he had received research grants from NIAAA, American Foundation for Suicide Prevention, and Takeda Pharmaceuticals. He received yearly compensation from Springer Publishing for acting as Editor-in-Chief of the Journal of Gambling Studies and had received royalties from Oxford University Press, American Psychiatric Publishing, Inc., Norton Press, and McGraw Hill. Dr. SRC consults for Cambridge Cognition and Shire.

\section{REFERENCES}

Abdi, H., \& Williams, L. J. (2013). Partial least squares methods: Partial least squares correlation and partial least square regression. Methods in Molecular Biology, 930, 549-579. doi:10.1007/978-1-62703-059-5_23

Aron, A. R., Fletcher, P. C., Bullmore, E. T., Sahakian, B. J., \& Robbins, T. W. (2003). Stop-signal inhibition disrupted by damage to right inferior frontal gyrus in humans. Nature Neuroscience, 6(2), 115-116. doi:10.1038/nn1003

Beebe, D. W. (2011). Cognitive, behavioral, and functional consequences of inadequate sleep in children and adolescents. Pediatric Clinics of North America, 58(3), 649-665. doi:10.1016/j.pcl.2011.03.002

Breslau, N., Roth, T., Rosenthal, L., \& Andreski, P. (1996). Sleep disturbance and psychiatric disorders: A longitudinal epidemiological study of young adults. Biological Psychiatry, 39(6), 411-418. doi:10.1016/0006-3223(95)00188-3

Casey, B. J., Heller, A. S., Gee, D. G., \& Cohen, A. O. (2017). Development of the emotional brain. Neuroscience Letters. Advance online publication. doi:10.1016/j.neulet.2017. 11.055

Chamberlain, S. R., \& Grant, J. E. (2018). Minnesota Impulse Disorders Interview (MIDI): Validation of a structured diagnostic clinical interview for impulse control disorders in an enriched community sample. Psychiatry Research, 265, 279-283. doi:10.1016/j.psychres.2018.05.006

Chee, M. W., \& Choo, W. C. (2004). Functional imaging of working memory after $24 \mathrm{hr}$ of total sleep deprivation. The Journal of Neuroscience, 24(19), 4560-4567. doi:10.1523/ JNEUROSCI.0007-04.2004

Choi, K., Son, H., Park, M., Han, J., Kim, K., Lee, B., \& Gwak, H. (2009). Internet overuse and excessive daytime sleepiness in adolescents. Psychiatry and Clinical Neurosciences, 63(4), 455-462. doi:10.1111/j.1440-1819.2009.01925.x

Corkum, P., Tannock, R., \& Moldofsky, H. (1998). Sleep disturbances in children with attention-deficit/hyperactivity disorder. Journal of the American Academy of Child and Adolescent Psychiatry, 37(6), 637-646. doi:10.1097/00004583199806000-00014

Drummond, S. P., Brown, G. G., Gillin, J. C., Stricker, J. L., Wong, E. C., \& Buxton, R. B. (2000). Altered brain response to verbal learning following sleep deprivation. Nature, 403(6770), 655-657. doi:10.1038/35001068

Ekinci, O., Celik, T., Savas, N., \& Toros, F. (2014). Association between Internet use and sleep problems in adolescents. Nöropsikiyatri Arşivi, 51(2), 122-128. doi:10.4274/npa.y6751

Frings, D. (2012). The effects of sleep debt on risk perception, risk attraction and betting behavior during a blackjack style gambling task. Journal of Gambling Studies, 28(3), 393-403. doi:10.1007/s10899-011-9266-9

Grant, J. E., Steinberg, M. A., Kim, S. W., Rounsaville, B. J., \& Potenza, M. N. (2004). Preliminary validity and reliability testing of a structured clinical interview for pathological gambling. Psychiatry Research, 128(1), 79-88. doi:10.1016/ j.psychres.2004.05.006

Höskuldsson, A. (1988). PLS regression methods. Journal of Chemometrics, 2(3), 211-228. doi:10.1002/cem.1180020306

Instanes, J. T., Klungsoyr, K., Halmoy, A., Fasmer, O. B., \& Haavik, J. (2018). Adult ADHD and comorbid somatic disease: A systematic literature review. Journal of Attention Disorders, 22(3), 203-228. doi:10.1177/1087054716669589

Jan, Y. W., Yang, C. M., \& Huang, Y. S. (2011). Comorbidity and confounding factors in attention-deficit/hyperactivity disorder and sleep disorders in children. Psychology Research and Behavior Management, 4, 139-150. doi:10.2147/PRBM. S14055

Johns, M. W. (1991). A new method for measuring daytime sleepiness: The Epworth Sleepiness Scale. Sleep, 14(6), 540-545. doi:10.1093/sleep/14.6.540

Kendall, A. P., Kautz, M. A., Russo, M. B., \& Killgore, W. D. (2006). Effects of sleep deprivation on lateral visual attention. The International Journal of Neuroscience, 116(10), 1125-1138. doi:10.1080/00207450500513922

Kessler, R. C., Adler, L., Ames, M., Demler, O., Faraone, S., Hiripi, E., Howes, M. J., Jin, R., Secnik, K., Spencer, T., Ustun, T. B., \& Walters, E. E. (2005). The World Health Organization Adult ADHD Self-Report Scale (ASRS): A short screening scale for use in the general population. Psychological Medicine, 35(2), 245-256. doi:10.1017/S0033291704002892

Kessler, R. C., Adler, L. A., Gruber, M. J., Sarawate, C. A., Spencer, T., \& Van Brunt, D. L. (2007). Validity of the World Health Organization Adult ADHD Self-Report Scale (ASRS) screener in a representative sample of health plan members. International Journal of Methods in Psychiatric Research, 16(2), 52-65. doi:10.1002/mpr.208

Killgore, W. D., Balkin, T. J., \& Wesensten, N. J. (2006). Impaired decision making following $49 \mathrm{~h}$ of sleep deprivation. Journal of Sleep Research, 15(1), 7-13. doi:10.1111/j.1365-2869. 2006.00487.x

Kubera, K. M., Schmitgen, M. M., Maier-Hein, K. H., Thomann, P. A., Hirjak, D., \& Wolf, R. C. (2018). Differential contributions of cortical thickness and surface area to trait impulsivity in healthy young adults. Behavioural Brain Research, 350, 65-71. doi:10.1016/j.bbr.2018.05.006

MacKillop, J., Weafer, J., Gray, J. C., Oshri, A., Palmer, A., \& de Wit, H. (2016). The latent structure of impulsivity: Impulsive choice, impulsive action, and impulsive personality traits. Psychopharmacology (Berl), 233(18), 3361-3370. doi:10.1007/ s00213-016-4372-0

McCoy, J. G., Tartar, J. L., Bebis, A. C., Ward, C. P., McKenna, J. T., Baxter, M. G., McGaughy, J., McCarley, R. W., \& Strecker, R. E. (2007). Experimental sleep fragmentation impairs attentional set-shifting in rats. Sleep, 30(1), 52-60. doi:10.1093/sleep/30.1.52

Pantelis, C., Barber, F. Z., Barnes, T. R., Nelson, H. E., Owen, A. M., \& Robbins, T. W. (1999). Comparison of set-shifting ability in patients with chronic schizophrenia and frontal lobe damage. Schizophrenia Research, 37(3), 251-270. doi:10.1016/S0920-9964(98)00156-X 
Parhami, I., Siani, A., Rosenthal, R. J., Lin, S., Collard, M., \& Fong, T. W. (2012). Sleep and gambling severity in a community sample of gamblers. Journal of Addictive Diseases, 31(1), 67-79. doi:10.1080/10550887.2011.642754

Patton, J. H., Stanford, M. S., \& Barratt, E. S. (1995). Factor structure of the Barratt Impulsiveness Scale. Journal of Clinical Psychology, 51(6), 768-774. doi:10.1002/10974679(199511)51:6<768::AID-JCLP2270510607>3.0.CO;2-1

Quinn, P. D., Stappenbeck, C. A., \& Fromme, K. (2011). Collegiate heavy drinking prospectively predicts change in sensation seeking and impulsivity. Journal of Abnormal Psychology, 120(3), 543-556. doi:10.1037/a0023159

Rogers, R. D., Tunbridge, E. M., Bhagwagar, Z., Drevets, W. C., Sahakian, B. J., \& Carter, C. S. (2003). Tryptophan depletion alters the decision-making of healthy volunteers through altered processing of reward cues. Neuropsychopharmacology, 28(1), 153-162. doi:10.1038/sj.npp.1300001

Schierenbeck, T., Riemann, D., Berger, M., \& Hornyak, M. (2008). Effect of illicit recreational drugs upon sleep: Cocaine, ecstasy and marijuana. Sleep Medicine Reviews, 12(5), 381-389. doi:10.1016/j.smrv.2007.12.004

Sheehan, D. V., Lecrubier, Y., Sheehan, K. H., Amorim, P., Janavs, J., Weiller, E., Hergueta, T., Baker, R., \& Dunbar, G. C. (1998).
The Mini-International Neuropsychiatric Interview (M.I.N.I.): The development and validation of a structured diagnostic psychiatric interview for DSM-IV and ICD-10. The Journal of Clinical Psychiatry, 59(Suppl. 20), 22-33; quiz 34-57.

Stein, M. D., \& Friedmann, P. D. (2005). Disturbed sleep and its relationship to alcohol use. Substance Abuse, 26(1), 1-13. doi:10.1300/J465v26n01_01

Van Veen, M. M., Karsten, J., \& Lancel, M. (2017). Poor sleep and its relation to impulsivity in patients with antisocial or borderline personality disorders. Behavioral Medicine, 43(3), 218-226. doi:10.1080/08964289.2017.1313719

Venkatraman, V., Chuah, Y. M., Huettel, S. A., \& Chee, M. W. (2007). Sleep deprivation elevates expectation of gains and attenuates response to losses following risky decisions. Sleep, 30(5), 603-609. doi:10.1093/sleep/30.5.603

Wong, M. M., Brower, K. J., \& Zucker, R. A. (2009). Childhood sleep problems, early onset of substance use and behavioral problems in adolescence. Sleep Medicine, 10(7), 787-796. doi:10.1016/j.sleep.2008.06.015

Young, K. (2009). Internet addiction: The emergence of a new clinical disorder. CyberPsychology \& Behavior, 1(3), 237-244. doi:10.1089/cpb.1998.1.237 